\section{Patient Attitudes Regarding ART and Insurance Coverage in the Military Health Care System}

\section{Abstract}

Objective: Assisted reproductive technology is an established infertility treatment which is not routinely covered by most health insurance programs including the health insurance program for the United States Department of Defense (DOD); TRICARE. The purpose of this study is to examine patient opinions regarding assisted reproductive services and insurance coverage in the military health care system.

Methods: An anonymous, randomly distributed cross-sectional survey of patients presenting for care at five different outpatient clinics was completed at Madigan Army Medical Center from February to May 2015. The surveys queried patients on insurance coverage for in-vitro fertilization. In addition, demographic data was obtained for each respondent. Chi-square, Student's t-test, and logistic regression were used for statistical analysis. A $P$ value of 0.05 was considered significant.

Results: 598 surveys were distributed with 527 completed and returned (88\%). $59 \%$ of respondents believe that TRICARE should cover some portion of advanced reproductive treatments, and $69 \%$ of respondents would support coverage even if there were an increased personal cost associated with providing this coverage regardless of whether they had a personal need for this service. In addition, more respondents from the infertility clinic (75\%) supported TRICARE coverage of advanced reproductive technology to those from other clinics (55\%). Respondents under age 40 (72\%) supported TRICARE insurance coverage of advanced reproductive technology compared to those ( $44.7 \%$ of respondents) aged 41 or greater.

Conclusion: Among DOD beneficiaries surveyed, a majority support insurance benefits for advanced reproductive technologies.

Keywords: Reproductive; Endocrinology; Infertility; Health
Scribner AN ${ }^{1}$, Hemman EA ${ }^{1}$, Heitmann RJ', Beesley RD', Burney $\mathrm{RO}^{2}$ and Gregory EC ${ }^{1 *}$

1 Department of Obstetrics and Gynecology, Madigan Army Medical Center, Tacoma, WA, USA

2 Department of Clinical Investigation, Madigan Army Medical Center, Tacoma, WA, USA

\section{*Corresponding author: Gregory EC}

gregory.e.chow.civ@mail.mil

Department of Obstetrics and Gynecology, Madigan Army Medical Center, Tacoma, WA, USA,

Tel: 2539682781

Citation: Scribner AN, Hemman EA, Heitmann RJ, Beesley RD, Burney RO, et al. (2018) Patient Attitudes Regarding ART and Insurance Coverage in the Military Health Care System. J Contracept Stud Vol.3 No.1:7

Received: December 12, 2017; Accepted: January 04, 2018; Published: January 11, 2018

\section{Introduction}

Infertility affects more than 7 million US women, or approximately $12 \%$ of the reproductive population [1] Assisted reproductive technology (ART), including in-vitro fertilization (IVF), has become an established and successful medical treatment for many types of infertility [2,3]. In 2014, 190,00 total ART cycles were performed in the United States (SART). On a more global scale, approximately 1.6 million cycles of IVF are undertaken each year resulting in more than 5 million children born as a result
[4]. Unfortunately, these numbers represent only a fraction of people who are affected by this disease both in the United States and around the world. Despite the increased use and success of this technology, patients who require advanced reproductive technologies to attain pregnancy continue to face significant financial challenges given the cost associated with these services.

Despite the classification of infertility as a disease by the World Health Organization in 2008 [5], the evaluation and treatment of infertility as a legitimate medical problem is not validated by 
routine coverage by most health insurance programs in the United States. This includes TRICARE, the health insurance program for the United States Department of Defense. A single cycle of IVF has been estimated to cost $\$ 12,400$ [6], which is the starting cost of a process which often requires multiple attempts in order to achieve a live birth. These high expenses are generally prohibitive to those couples who require this therapy to reproduce.

Several studies have demonstrated that the high cost associated with IVF excludes many couples from receiving treatment $[7,8]$ while also leading to an increase in multi-embryo transfer rates, ultimately leading to a higher incidence of adverse outcomes associated with multiple gestations [9]. Coverage of these services by health insurance may improve access to care and promote safer practices $[10,11]$. Several states have passed insurance mandates requiring insurance companies to cover some of the costs associated with IVF. Previous studies comparing states with and without mandated insurance coverage for IVF demonstrated lower rates of multiple gestation, increase in access to these services, albeit with lower pregnancy rates [12].

At this time, no studies have been published evaluating patient opinions and attitudes in a military regarding insurance coverage of advanced reproductive technology. The purpose of this study is to examine patient attitudes towards advanced reproductive services and insurance coverage in the Military Health care System (MHS) and evaluate patient factors that may be correlated with these attitudes.

\section{Materials and Methods}

This study was conducted at Madigan Army Medical Center (MAMC) in Tacoma, WA, a teaching hospital and referral center serving all surrounding military instillations for the pacificnorthwest region of the country. We conducted a survey study from February 2015 to May 2015 to evaluate patient attitudes toward advanced reproductive technology and insurance coverage. This study was approved by the Madigan Army Medical Center Institutional Review Board.

The survey instrument was developed by the authors and pilot tested on a convenience sample of patients $(n=25)$ to assess clarity and comprehension of the survey. The survey elicited demographic information including age, gender, race/ethnicity, education level, annual income, relationship status and number of children. The survey also asked the respondents hypothetical questions regarding their opinions about insurance coverage of IVF services and different scenarios that coverage may apply to (Appendix A).

Survey packets were prepared containing a summary welcome letter explaining the study and the survey form. Surveys were color coded based on the clinic distribution. Each survey was numbered sequentially to track survey distribution and rates of return. The surveys were randomly handed out to TRICARE beneficiaries, seeking care at five MAMC outpatient clinics. Obstetrics/Gynecology; Reproductive Endocrinology and Infertility (RE/I); General Surgery; Family Practice; and Pediatrics. Patients were requested to voluntarily participate when they presented for their clinic appointment. After completion of the survey, patients were instructed to return their surveys to locked boxes located within the waiting room of each clinic. Surveys were collected until 80 completed surveys had been collected from each respective clinic; a representative sampling from each clinical area.

The primary outcome measure was to solicit the opinions of patients regarding the question of whom should pay for IVF services. This was assessed by the following question, "In-vitro fertilization results in pregnancy in 4 out of 10 couples who have this service. It is currently not covered by medical insurance. One attempt at pregnancy can cost $\$ 10,000-\$ 15,000$. In your opinion, whom do you think should pay for this procedure for the military population?" Patients had the option to choose one of four responses to this question (Appendix $A$, see question $2 a$ ).

Patients that supported insurance coverage of IVF were asked about their opinions regarding their support even if providing ART coverage increased insurance costs to all members. Several other questions were posed which included questions regarding coverage for single individuals, same sex couples, and how many attempts insurance should cover.

The data from the surveys was compiled into an electronic spreadsheet using SPSS version 22 (SPSS Inc. 2013, Chicago, IL, USA). Descriptive statistics were utilized to evaluate frequencies, means, and proportions with standard deviations. Chi-square and independent t-test were used to analyze for differences between basic demographic data by groups as specified on the questionnaire. Finally, multivariate analysis by multiple multinomial logistic regression was employed to determine patient factors that were significantly associated with the primary and secondary outcomes that have multiple categories. A P value of $<0.05$ was considered significant.

\section{Results}

A total of 598 surveys were administered during the study period with 527 completed and returned for an overall response rate of 88.1\% (Figure 1).

The margin of error of a survey can be expressed as, $(1 / \sqrt{n})$ $\mathrm{n}=$ number of respondents to the survey. With a return of 527 surveys, the margin of error with this population is 0.04 , within statistical significance for the population surveyed. The RE/I clinic had the highest response rate at $95.7 \%$, followed by Pediatrics (94.4\%) and General Surgery (92.6\%).

The demographics for respondents completing the questionnaire are shown in (Table 1 ). We expectedly found a statistically significant difference in gender, age, relationship status and number of children across surveys collected in different clinics. As anticipated, respondents from the OB/GYN clinic, RE/I clinic and Pediatrics clinic were primarily female. The majority of respondents were between ages 23 and 40, although $59 \%$ of the respondents from the general surgery clinic were older than 51 years of age. Respondents from the RE/I clinic were more likely to have 0 children (64.5\%) than those from other clinics. 


\section{8 surveys distributed}

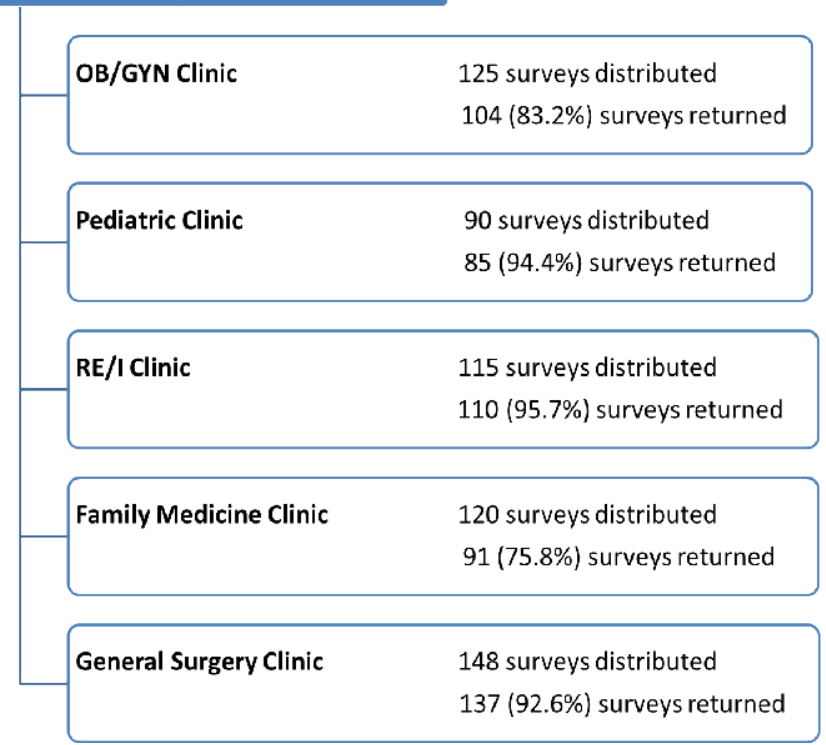

Figure 1 A total of 598 surveys were administered during the study period with 527 completed and returned for an overall response rate of $88.1 \%$.

When queried as to whom should pay for advanced reproductive services only $9.9 \%$ of participants answered that the patient should be responsible. $51.2 \%$ participants answered that TRICARE military healthcare should be the payer. In contrast, only $4.2 \%$ believe that private health care should pay for these services. The remainder of respondents (34.7\%) was undecided or may have had an alternative opinion regarding payment for ART.

The opinions regarding coverage were significantly disparate when considering gender and age of the respondents]. Females were more likely to favor coverage by TRICARE (69.8\%) whereas only $(44.8 \%)$ males were of the same opinion, $(P<0.001)$. As well, younger individuals were more likely to favor coverage by TRICARE. Of those aged $18-22,76.7 \%$ of respondents stated that TRICARE should cover these benefits compared to only $35.0 \%$ of those aged $>65,(P<0.001)$.

The majority of patients answered favorably when questioned about whether increasing insurance costs for all patients were acceptable in order to accommodate the relatively small number of patients requiring IVF. Not surprisingly, patient income was statistically different between those answering yes and those responding no to that question. Patients with higher incomes were more likely to support a relative increase in premiums to support ART than those of lower socioeconomic strata $(P<0.01)$. $73 \%$ of respondents with annual incomes greater than $\$ 70,000$ supported insurance increases compared to $55 \%$ of those households earning less than $\$ 20,000$ and $60 \%$ of households earning between $\$ 20,000-29,000$.

In contrast, opinions regarding IVF coverage for single individuals is not as clear. Only $31 \%$ of respondents supported IVF coverage for single individuals, $30 \%$ of participants did not support coverage, and $39 \%$ of people did not respond to this question.
When asked how many attempts, or cycles, should be covered, $44.1 \%$ of respondents answered $1-2$ cycles, $21.4 \%$ answered $3-4$ cycles, $4.5 \%$ answered $5-10$ cycles, $2.0 \%$ stated greater than 10 cycles. $6.7 \%$ of respondents felt that no cycles should be covered. Results were statistically different $(P=0.004)$ when responses from the RE/I clinic were compared to respondents from all other clinics. Respondents from the RE/I clinic were more likely to support an increasing number of attempts than respondents from all other clinics.

\section{Discussion}

The value of ART as an effective treatment for infertility is undisputed. Unfortunately, cost is a considerable barrier which ultimately dissuades many couples from realistically considering its use in their infertility journey. The military medical system provides ART services at 7 centers throughout the United States. While there is some variance of the price of an IVF cycle between the centers; in all cases, the military beneficiary is required to pay out of pocket costs $(\$ 5-8000)$ for each cycle undertaken.

In this study, examination of the opinions of military healthcare beneficiaries toward insurance coverage of advanced reproductive technology suggest that a majority of those completing this survey favor insurance coverage of this service. The opinions expressed represent a wide sampling of beneficiaries who access the military medical system. Specifically, this sentiment expressed is clearly directed toward the military medical insurance program (TRICARE) and not private insurance carriers. Of course, an inherent bias in the response to this query is likely as most military beneficiaries carry no competing private insurance and thereby have no viable alternative coverage. In addition, we fully recognize that expression of an opinion and the reality of the personal impact which comes with a change in policy may not be completely comprehended for each individual respondent.

We sought to obtain a diverse cohort of patients throughout the military facility which included the spectrum of patients with the most vested interested in fertility care (RE/I clinic) to those with perhaps very little interest in fertility treatments (general surgery clinic). The other departments (OB/GYN, Family Medicine, Pediatrics) were selected as they represented more common primary care portals through which a large diverse patient population access their medical care with a wide breadth of medical issues ranging from health maintenance visits to acute medical problems.

Survey response return rate was excellent in all departments. While the surveys were distributed "randomly" to patients presenting for daily appointments, there was undoubtedly selection bias introduced which may have been affected by a diverse group of variables ranging from the day of the week the surveys were distributed to the reception clerks distributing the surveys. Additionally, participation bias may play a role in skewing the data shown as completion of the survey was entirely voluntary and not required. The general altruistic nature of patients in the hospital setting may be different than those encountered in an alternative setting. Still, 527 returned surveys 
Table 1 Demographics of patients completing the survey stratified by clinic.

\begin{tabular}{|c|c|c|c|c|c|c|}
\hline & Family medicine & General surgery & OB/GYN & Pediatrics & $\operatorname{Re} / \mathrm{I}$ & $P$ value \\
\hline \multicolumn{7}{|l|}{ Gender } \\
\hline Male & $41(45.1)$ & $57(41.6)$ & $1(1.0)$ & $9(10.6)$ & $5(4.5)$ & \\
\hline Female & $50(54.9)$ & $80(58.4)$ & $104(99.0)$ & $76(89.4)$ & $105(95.5)$ & $<0.001$ \\
\hline \multicolumn{7}{|l|}{ Race } \\
\hline White & $66(72.5)$ & $96(71.6)$ & $64(62.1)$ & $61(71.8)$ & $78(70.9)$ & \\
\hline Black or African American & $14(15.4)$ & $15(11.2)$ & $13(12.6)$ & $7(8.2)$ & $5(4.5)$ & \\
\hline Asian & $3(3.3)$ & $10(7.5)$ & $6(5.8)$ & $8(9.4)$ & $9(8.2)$ & \\
\hline Native Hawaiian or other pacific islander & $3(3.3)$ & $10(7.5)$ & $6(5.8)$ & $8(9.4)$ & $9(8.2)$ & \\
\hline American Indian/Alaaskan native & $1(1.1)$ & $2(1.5)$ & $0(0.0)$ & $2(2.4)$ & $3(2.7)$ & 0.084 \\
\hline \multicolumn{7}{|l|}{ Ethnicity } \\
\hline Hispanic & $8(8.8)$ & 15 (11.4) & $18(17.6)$ & $11(12.9)$ & $13(11.9)$ & \\
\hline Not Hispanic & $83(91.2)$ & $117(88.6)$ & $84(82.4)$ & $74(87.1)$ & $96(88.1)$ & 0.432 \\
\hline \multicolumn{7}{|l|}{ Age } \\
\hline $18-22$ & $5(5.5)$ & $1(0.8)$ & $10(9.6)$ & $4(4.7)$ & $10(9.1)$ & \\
\hline $23-30$ & $27(29.7)$ & $14(10.8)$ & $55(52.9)$ & $47(55.3)$ & $57(51.8)$ & \\
\hline $31-40$ & $22(24.2)$ & $22(16.9)$ & $27(26.0)$ & $30(35.3)$ & $39(35.5)$ & \\
\hline $41-50$ & $13(14.3)$ & $17(13.1)$ & $8(7.7)$ & $4(4.7)$ & $3(2.7)$ & \\
\hline $51-65$ & $0(0.0)$ & $55(42.3)$ & $3(2.9)$ & $0(0.0)$ & $1(0.9)$ & \\
\hline$>65$ & $0(0.0)$ & $21(16.2)$ & $1(1.0)$ & $0(0.0)$ & $0(0.0)$ & $<0.001$ \\
\hline \multicolumn{7}{|l|}{ Education } \\
\hline Elementary only & $0(0.0)$ & $1(0.7)$ & $0(0.0)$ & $0(0.0)$ & $0(0.0)$ & \\
\hline Some high school & $0(0.0)$ & $6(4.5)$ & $1(1.0)$ & $0(0.0)$ & $2(1.8)$ & \\
\hline High school graduate, GED or & $14(15.4)$ & $18(13.4)$ & $21(20.4)$ & $11(12.9)$ & $16(14.5)$ & \\
\hline Some college & $27(29.7)$ & $55(39.6)$ & $45(43.7)$ & $33(38.3)$ & $45(40.9)$ & \\
\hline College degree or higher & $49(53.8)$ & $36(35.0)$ & $36(35.0)$ & $41(48.2)$ & $47(42.7)$ & 0.241 \\
\hline \multicolumn{7}{|l|}{ Income (annual) } \\
\hline$<\$ 20,000$ & $4(4.5)$ & $5(4.1)$ & $4(4.0)$ & $3(3.7)$ & $4(3.7)$ & \\
\hline$\$ 20,000-\$ 29,999$ & $11(12.5)$ & $12(9.9)$ & $25(25.0)$ & $13(16.0)$ & $16(14.8)$ & \\
\hline$\$ 30,000-\$ 39,000$ & $10(11.4)$ & $21(17.4)$ & $20(20.0)$ & $14(17.3)$ & $24(22.2)$ & \\
\hline$\$ 40,000-\$ 49,000$ & $10(11.4)$ & $13(10.7)$ & $9(9.0)$ & $12(14.8)$ & $14(13.0)$ & \\
\hline$\$ 50,000-\$ 59,000$ & $10(11.4)$ & $19(15.7)$ & $11(11.0)$ & $9(11.1)$ & $16(14.8)$ & \\
\hline$\$ 60,000-\$ 69,000$ & $11(121.5)$ & $9(7.4)$ & $5(5.0)$ & $7(8.6)$ & $4(3.7)$ & \\
\hline$\$ 70,000-\$ 79,000$ & $4(4.5)$ & $9(7.4)$ & $9(9.0)$ & $5(6.2)$ & $7(6.5)$ & \\
\hline$>\$ 80,000$ & $28(31.8)$ & $33(27.3)$ & $17(17.0)$ & $18(22.2)$ & $23(21.3)$ & 0.433 \\
\hline \multicolumn{7}{|l|}{ Relationship status } \\
\hline Married/partnered & $73(80.2)$ & $110(82.1)$ & $92(88.5)$ & $77(90.6)$ & $108(98.2)$ & \\
\hline Divorced & $8(8.8)$ & $9(6.7)$ & $6(5.8)$ & $4(4.7)$ & $1(0.9)$ & \\
\hline Separated & $1(1.1)$ & $4(3.0)$ & $1(1.0)$ & $2(2.4)$ & $0(0.0)$ & \\
\hline Widowed & $2(2.2)$ & $6(4.5)$ & $1(1.0)$ & $0(0.0)$ & $0(0.0)$ & \\
\hline Never married & $7(7.7)$ & $5(3.7)$ & $4(3.8)$ & $2(2.4)$ & $1(0.9)$ & 0.018 \\
\hline \multicolumn{7}{|l|}{ Children } \\
\hline 0 children & $32(35.6)$ & $12(9.0)$ & $31(29.8)$ & $0(0.0)$ & $71(64.5)$ & \\
\hline 1 child & $13(14.4)$ & 20 (14.9) & $33(31.7)$ & $33(38.8)$ & $23(20.9)$ & \\
\hline 2 children & $20(22.2)$ & $45(33.6)$ & $23(22.1)$ & $35(41.2)$ & $11(10.0)$ & \\
\hline 3 or more children & $25(27.8)$ & $57(42.5)$ & $17(16.3)$ & $17(20.0)$ & $5(4.5)$ & \\
\hline
\end{tabular}

represent a representative sample of a general population with a margin of error of 0.04 .

As expected, those with the most vested interest (RE/I clinic) had the highest rate of return for this survey and expressed opinions with the greatest desire for insurance coverage. However, even in those clinics with patients who may have been removed from the reproductive process (i.e,. general surgery), there was considerable support for such an initiative. The overall return rate for all clinics were very high which seem to suggest amongst the population surveyed that there was a significant interest in this topic of discussion.

\section{Conclusion}

In summary, the overall opinion of military medical beneficiaries supports insurance coverage by the military medical system (TRICARE) for IVF services. This opinion was present even in the 
context of increasing costs to the individual and for those who do not utilize or have the need for such services.

\section{References}

1 Abma JC, Chandra A, Mosher WD, Peterson LS, Piccinino LJ (1997) Fertility, family planning, and women's health: new data from the 1995 National Survey of Family Growth. Vital Health Stat 23: 1-114.

2 Cramer DW, Liberman RF, Powers D, Hornstein MD, McShane P, et al. (2000) Recent trends in assisted reproductive techniques and associated outcomes. Obstet Gynecol 95: 61-66.

3 Jain T, Missmer SA, Hornstein MD (2004) Trends in embryo-transfer practice and in outcomes of the use of assisted reproductive technology in the United States. N Eng J Med 350: 1639-1645.

4 ESHRE (2012) The European IVF-monitoring Consortium, for the European Society of Human Reproduction Embryology (ESHRE), assisted reproductive technology in Europe, 2007: results generate from European registers by ESHRE. Hum Reprod 27: 954-966.

5 Zegers-Hochschild F, Adamson GD, de Mouzon J, Ishihara O, Mansour R, et al. (2009) International Committee for Monitoring Assisted Reproductive Technology (ICMART) and the World Health Organization (WHO) revised glossary of ART terminology. Fertil Steril 92: 1520-1524.

\section{Acknowledgements}

The authors would like to acknowledge Dr. Raywin Huang for his assistance with statistical data analysis.

6 American Society for Reproductive Medicine. Is In Vitro Fertilization Expensive? 2013.

7 Jain T, Hornstein MD. To pay or not to pay. Fertil Sterl 80: 27-29.

8 Gleicher N, Vanderlaan B, Karande V, Pratt D, Morris R et al (1996) Infertility treatment dropout and insurance coverage. Obstet Gynecol 88: 289-293.

9 Chambers GM, Adamson GD, Eijkemans MJ (2013) Acceptable cost for the patient and society. Fertil Steril 100: 319-327.

10 Martin JR, Bromer JG, Sakkas D, Patrizio P (2011) Insurance coverage and in vitro fertilization outcomes: a US perspective. Fertil Steril 95: 964-969.

11 Chambers GM, Hoang VP, Illingworth PJ (2013) Socioeconomic disparities in access to ART treatment and the differential impact of a policy that increased consumer costs. Hum Reprod. 28: 3111-3117.

12 Martin JR, Bromer JG, Sakkas D, Patrizio P (2011) Insurance coverage and in vitro fertilization outcomes: a U.S. perspective. Fertil Steril 95: 964-969. 